\title{
Blueprint and Implementation of auto motion observation exploitation GSM/GPS technology with cellular device
}

\author{
V. Surekha (M.Tech), Mr M.Lakshmipathy, \\ Kuppam Engineering College, Kuppam, Chittoor Dist, A.P, India \\ Asst.Professor, Dept. of ECE Kuppam Engineering College, Kuppam
}

\begin{abstract}
:
An effective vehicle following framework is made public and dead for following the event of any ready vehicle from any space whenever. The projected framework created nice utilization of a acknowledge innovation that consolidates a sensible phone application with a microcontroller. This may be something however tough to form and low cost contrasted with others. The printed in-vehicle gismo works utilizing world Positioning System (GPS) and world System for mobile Communication (GSM) innovation that's a standout amongst the foremost widely known routes for vehicle following. The gismo is planted within a vehicle whose position is to be resolved and followed more and more. A microcontroller is used to regulate the GPS and GSM/GPRS modules. The vehicle following framework utilizes the GPS module to induce geographic directions at time interims. The GSM module is used to transmit and refresh the vehicle space to a information. A Smartphone application is in addition created for systematically checking the vehicle space.
\end{abstract}

Index Terms: Microcontroller, GPS, GSM/GPRS Technology, Smartphone application.

\section{INTRODUCTION}

Vehicle following frameworks were at first bestowed for the delivery/load enterprises since people ought to recognize the vehicle space at no matter purpose they needed. Presently a-days the innovation is changing into fast a robotized following of the vehicle framework is being used as an area of Associate in Nursing assortment of approaches to trace and show the world of the vehicle. During this manner, shoppers can have the capability to perpetually screen a moving vehicle for the asking utilizing the Smartphone application and judge the evaluated separation and time for the vehicle to the touch base at a given goal. With a particular finish goal to demonstrate the chance and viability of the framework, this paper presents check aftereffects of the vehicle following framework and a couple of encounters on all the way down to earth executions. This paper proposes a vehicle following framework utilizing the GPS and GSM innovations and advanced itinerant to grant higher administration and smallest effort for the shopper.

\section{Existing System:}

The main downside of this system is just monitor the thieving vehicle and grievance to the department of local government then they take action against grievance. It takes lasting to gets their vehicle back. This technique primarily approach in four wheeler. Though its GPS enabled vehicle, we will solely track and monitor the vehicle. Many security and pursuit systems area unit designed to help firms with sizable amount of vehicles and a number of other usage functions. They can't allow the owner to speak with the vehicle on-line, though the owner is definite that his vehicle was purloined.

\section{Proposed System:}

The point planned is to execute smaller than traditional model by utilizing a solitary chip microcontroller within the vehicle. GPS are going to be settled within the vehicle to screen and to get space of the vehicle. With the help of the GPS esteem, the separation will computed as for time. The course and therefore the separation at bolstered into the microcontroller which are going to be transmitted to GSM through advanced adjustment procedures. At the beneficiary finish the flag are going to be distinguished and demodulated with computerized reception strategy. At that time the flag are going to be given to automaton moveable.

\section{System Specifications}

A. Hardware Requirements

1. Microcontroller ARM7

2. Power Supply

3. 16X2 LCD

4. GSM

5. GPS

6. L293D Motor Driver
B. Software Requirements
1. Embedded C
2. Keil Software 


\section{Flash Magic}

3. Working

Block Diagram:

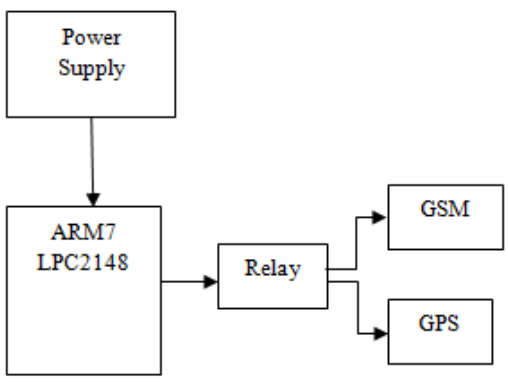

Figure 3.1 Block Diagram

ARM7

"ARM" is that the abbreviation of "Advanced reduced instruction set computing Machines". It is a widespread processor cores within the world. It's particularly employed in transportable devices thanks to affordable performance and low power consumption. ARM could be a family of reduced instruction set computing architectures. The fig. of ARM is shown within the fig9. The raise 16/32-bit ARM7TDMI-S microcontroller coaching board is extremely specifically designed to assist students to fulfill their needed skills within the space of embedded systems. it's attainable to style the kit in such manner that everyone the vital options of the microcontroller are going to be fully utilized by the scholars.

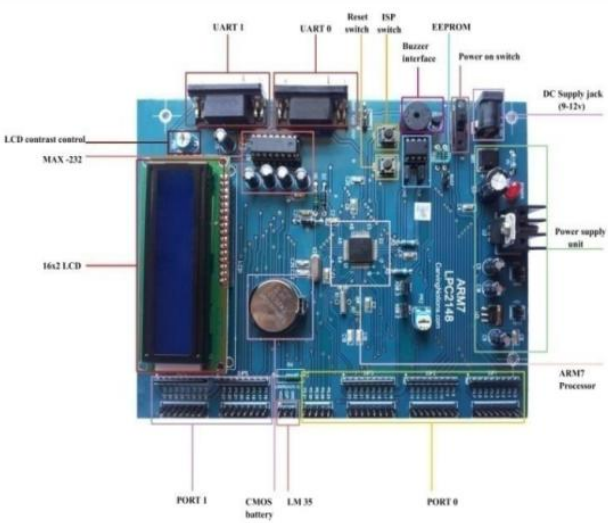

Figure 3.2 Hardware System

The kit can supports in system programming (ISP) that is finished by interface. Raise Board has advanced and new choices that help the user in implementing advanced logic, that area unit utilized in the planning of Embedded Systems. Pipeline techniques area unit with success utilized so all elements of the memory and process systems will perform unceasingly. Typically, once one instruction is being dead, its successor is decoded, and a 3rd instruction is fetched from memory.
The ARM7 may be a general purpose 32bit silicon chip, that offers high performance and extremely low power consumption. The ARM design is predicated on Reduced Instruction Set pc (RISC) principles, and also the instruction set and connected decipher mechanism a lot of easier than those of small programmed complicated Instruction Set Computers (CISC). This simplicity ends up in a high instruction turnout and spectacular time period interrupt response from a little and efficient processor core.

\section{Power Supply}

A power offer may be a device that delivers a precise voltage to a different device as per its wants. There square measure several power provides on the market nowadays within the market like regulated, unregulated, variable etc, and also the call to select the proper one depends entirely on what device you're making an attempt to control with the facility offer. Power provides, typically referred to as power adapters, or just adapters, square measure on the market in numerous voltages, with variable current capacities, that is nothing however the utmost capability of an influence offer to deliver current to a load (Load is that the device you're making an attempt to produce power to).

Here is the circuit diagram for the Power Supply:

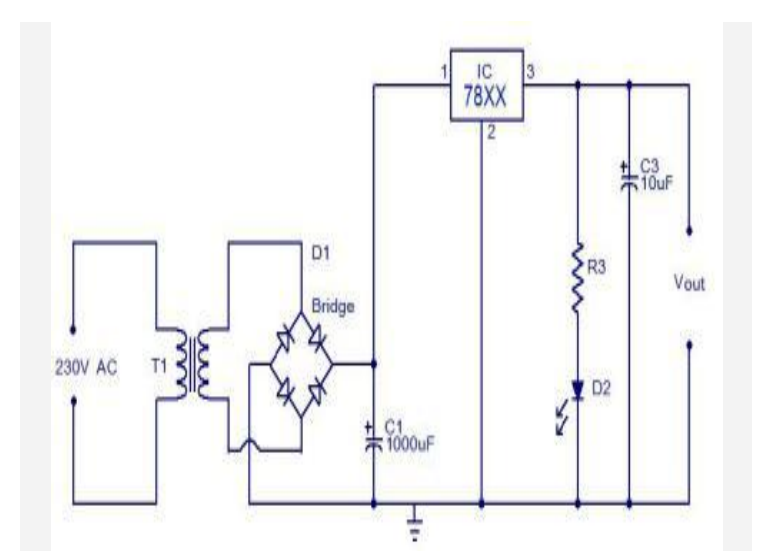

Figure 3.3 Circuit Diagram

\section{Working of Power Supply:}

The AC mains area unit fed to the electrical device those steps down the 230 Volts to the required voltage. The bridge rectifier follows the electrical device so changing $\mathrm{AC}$ voltage into a DC output and thru a filtering electrical condenser feeds it directly into the input (Pin 1) of the transformer. The common pin (Pin 2) of the transformer is grounded. The output (Pin 3) of the transformer is 1st filtered by a electrical condenser, so the output is taken. Make the circuit on a general purpose $\mathrm{PCB}$ and use a two Pin (5A) plug to attach 
the electrical device input to the $\mathrm{AC}$ mains via insulated copper wires.

Relay:

A relay may be represented in broad Associate in Nursing general terms as an electrically operated switch. several relays use Associate in Nursing magnet to work a switch automatically. Relays square measure used wherever many circuits should be controlled by one signal. They will even be created helpful wherever it's needed to regulate a circuit by a coffee hoppedup signal.

\section{GSM/GPRS9600:}

Global system for mobile communication (GSM) could be a globally accepted customary for digital cellular communication. GSM is that the name of a customarization cluster established in 1982 to make a standard European mobile phoneto-phone standard that will formulate specifications for a pan-European mobile cellular radio system operative at 900 megahertz. it's calculable that a lot of countries outside of Europe can be part of the GSM partnership.

\section{GSM Network:}

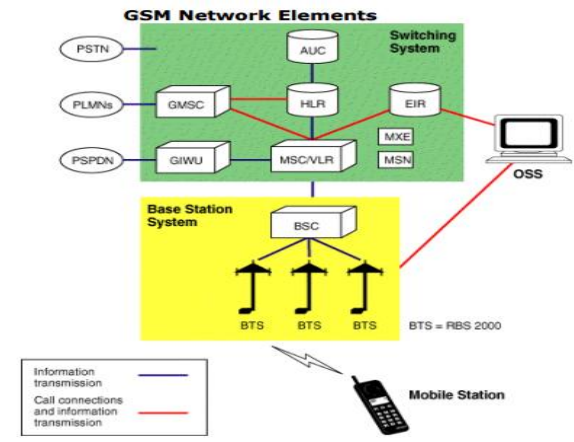

Figure 3.4 GSM Network Elements

GPS:

GPS is employed in vehicles for each trailing and navigation. Trailing systems modify a base station to stay track of the vehicles while not the intervention of the driving force wherever, as navigation system helps the driving force to succeed in the destination. Whether or not navigation system or trailing system, the design is additional or less similar. Once an accident occurred in anywhere then GPS system tracks the position of the vehicle and sends the knowledge to the actual person through GSM by alerting the person through SMS or by a decision.

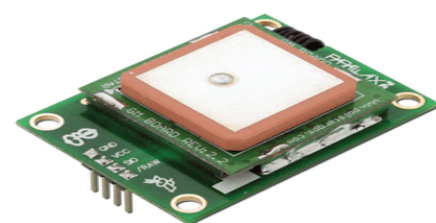

Figure 3.5 GPS System

\section{RESULTS}

The GPS module receives the geographic coordinates from the satellite. The microcontroller read the vehicle location information from GPS module. A sim will be inserted into the GSM module. The vehicle's location information then transmitted to the web server through GSM/GPRS network.

The below figure shows the hardware connections and after giving the connections dump the code into microcontroller by using USB cable and power on the supply. after giving connections LCD will initialize and GPS,GSM all modules will initialize.

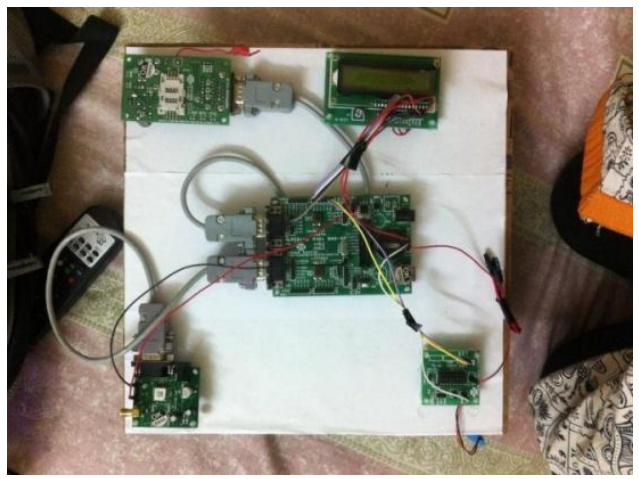

The below figure shows after giving the power supply the GPS and GSM modules will initialize and it will wait for message from the user, after receiving the message GPS will send the longitude and latitude values to the user.

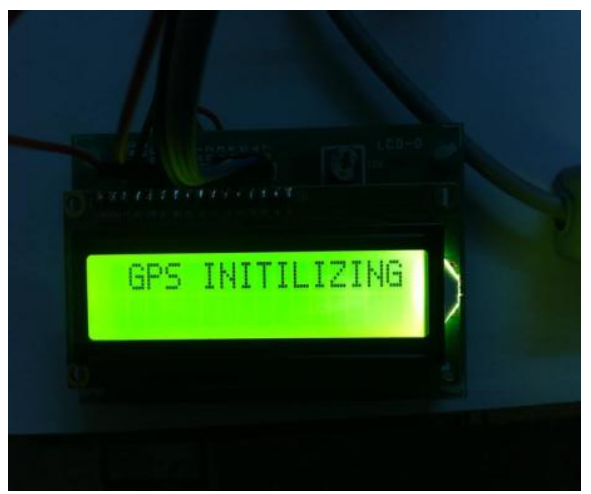


The below figure shows the message which is received from the GPS module.Here the message consists of the latitude and longitude values. By sending the message $\$ 2$ we can start the engine and by sending the message $\$ 3$ we can stop the engine.

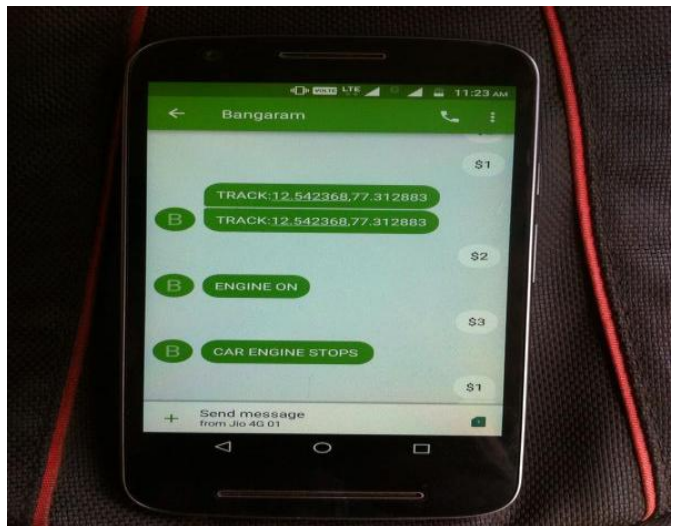

The below figure shows the exact location of the vehicle in the developed smartphone application. By using this application we can track the vehicle location anytime.

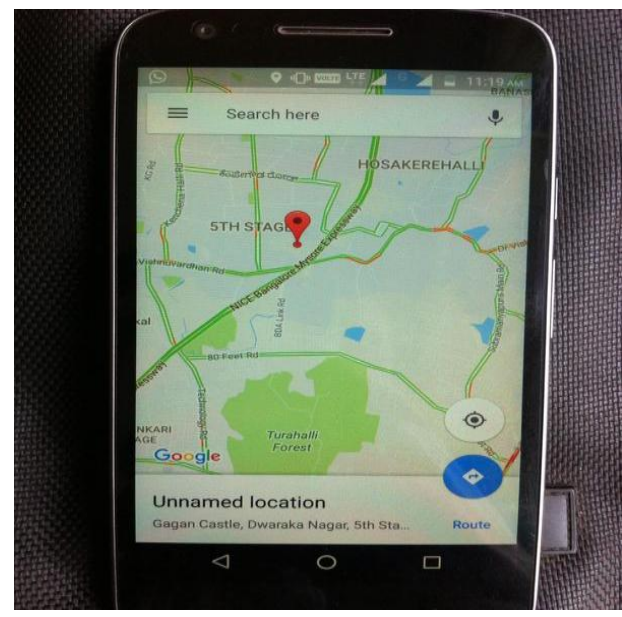

Applications:

- Used in buses

- Used in cars

- Used in automated vehicles Advantages:

- Lost vehicle can be tracked

- Accident occurred vehicle can be tracked

\section{CONCLUSION}

Outline and Implementation of car following and perceptive utilizing GPS and ARM processors to remain far away from vehicle crash and reduce automobile overloads out and concerning within the meanwhile speed of the vehicle in addition checked. The created framework adequately screens and vehicle moving. The frameworks are often reached out for checking the complete parameters like fuel, fuel go, speed, temperature, oil in tank. The perceptive frameworks are custom-built with completely different module interfaces affordable for shoppers of shifting capability associate degreed for master shoppers to such an extent that the framework are often preserved effortlessly and communicated with simply. This framework is efficacious in abundant application, as an example, observation, security following, which could be introduced in mining trucks, payload trucks, autos, cruiser, and robot. The frameworks are often useful for a few applications.

\section{REFERENCES}

[1]. NJ.-P. Hubaux, S. C Apkun, and J. Luo "The security and privacy of smart vehicles", IEEE security and Privacy magazine, vol 2.no3, pp 49-55, 2004.

[2]. Hui Hu, LianFang, published a paper title "design and Implementation of vehicle monitoring system based on GPS/GSM/GIS", Third International symposium on Intelligent information Technology Application 2009.

[3]. Mohammad A. Al-Khedher, "Hybrid GPS-GSM Localization of Automobile Tracking System", International Journal of Computer Science \& Information Technology (IJCSIT) Vol 3, No 6, Dec 2011

[4]. Fan, X., W. Xu, H. Chen, and L. Liu, "CCSMOMS:A Composite Communication Scheme for Mobile Object Management System",20th International Conference on Advanced Information Networking and Applications, Volume 2, Issue 18-20, April 2006,pp. 235-239.

[5]. Aaron Smith, "Nearly half of American adult are Smart phone", http://pewinternet.org/ /media/Files/Repo rts/2012/Smartphone\%20ownership\%201 2.pdf,2012

[6]. Internet Sources

[7]. www.gsmworld.com,october 2008.

[8]. www.google.com.

[9]. www.gnokii.org,november 2008.

[10]. www.keil.com,november 2009.

[11]. http://www.positronindia.in/datasheet/Dat a sheets $\operatorname{sim} 300$

[12]. Ambade Shruti Dinker and S.A Shaikh,"Design and Implementation of vehicle tracking system using GPS",Journal of Information Engineering and Applications, Vol 1,No.3,2011 